\title{
Comparison of Muscle Energy Technique vs Mulligan to Improve Gird in District Level Cricket Bowlers
}

\author{
Kimaya Vinod Kini ${ }^{1}$, Deepali Rathod ${ }^{2}$, Ajay Kumar ${ }^{3}$ \\ ${ }^{1}$ Graduate, DPO's Nett College of Physiotherapy, Thane, India \\ ${ }^{2}$ Assistant Professor, TMVs Lokmanya Medical College of Physiotherapy, Kharghar, India \\ ${ }^{3}$ Principal, DPO's Nett College of Physiotherapy, Thane, India \\ Corresponding Author: Kimaya Vinod Kini
}

\begin{abstract}
Background: Cricket bowlers often have to perform overhead throwing due to which in the dominant arm, compared with the nondominant arm the magnitude of glenohumeral internal rotation is decreased and magnitude of external rotation is increased. Glenohumeral internal rotation deficits (GIRD) are deficits of internal rotation and total arc of motion in the dominant side which is common in overhead athletes including cricket bowlers. Hence the aim of the study was to compare the effectiveness of muscle energy technique vs mulligan in patients with glenohumeral internal rotation deficit.
\end{abstract}

Methodology: 30 patients were included in the study which was divided into two groups; Group A with 15 patients and Group B with 15 patients.30 cricket bowlers with presence of glenohumeral internal rotation deficit were selected. Before application of muscle energy technique and mulligan active shoulder range of motion using full scale goniometer was taken. Treatment was given for 4 weeks, 6 sessions per week. All patients were given hotpacks and stretching for internal rotators before treatment. The difference of pre and post was then calculated. The data was statistically analysed using paired t-test.

Results: Mulligan mobilization and met are both effective in improving glenohumeral internal rotation deficit in 4 weeks.

Conclusion: Mulligan mobilization and met helps to improve glenohumeral internal rotation deficit almost with same margin.

Keywords: cricket bowlers, Glenohumeral internal rotation deficit, mulligan, Met

\section{INTRODUCTION}

Cricket is a popular sport that requires a combination of physical fitness, skill and strategy. Although a non-contact sport, overuse and impact injuries are common since players do a wide variety of physical activities, including running, throwing, batting, bowling, catching and diving. (1)

There are $60 \%$ fast bowlers experiencing cricket-related musculoskeletal problems, in which there is $16 \%$ prevalence of shoulder problems. ${ }^{(2)}$
Throwing is a whole body activity that commences with drive from the large leg muscles and rotation of the hips and progresses through segmental rotation of the trunk and shoulder girdle. Throwing can be divided into four phases:-

1. Preparation / Wind-up.

2. Cocking.

3. Acceleration.

4. Deceleration / Follow-through. ${ }^{(3)}$

The cocking phase is the high torque phase with maximal shoulder external rotation. The associated pathology that takes 
place during this phase is internal impingement and GIRD (glenohumeral internal rotation deficit).

Glenohumeral internal rotation deficit (GIRD) is an adaptive process in which the throwing shoulder experiences a loss of internal rotation (IR). Currently, GIRD can be defined by a loss of $>20$ degree of internal rotation compared to the contralateral shoulder. ${ }^{(4)}$

One of the factors of glenohumeral joint arthrokinematics is to allow optimal glenohumeral rotation, which includes both internal and external rotations. If there is proper balance of internal and external rotation, it allows the humeral head to remain centered in the glenoid fossa. Many studies have shown that in the dominant arm, compared with the nondominant arm, the magnitude of glenohumeral internal rotation is decreased and magnitude of glenohumeral external rotation is increased in most throwing athletes. Adaptive changes to bone and soft tissue occur as a result of the repetitive throwing motion that contributes to the presence of GIRD in the overhead athletes. GIRD is one of the most important factors influencing injury risk in the arm. ${ }^{(5)}$

There are various techniques proved to be effective in improving GIRD such as stretching, muscle energy technique, Maitland, Mulligan etc.

"Muscle energy technique (MET) are form of soft tissue, or joint manipulations or mobilizations, deriving from osteopathic medicine, employed in the treatment of musculoskeletal dysfunction" according to Leon Chaitow Muscle Energy Technique (4th edition).

Muscle energy technique is given by asking the subject to create a force by activating the targeted muscle tendon unit against a counterforce applied by the researcher, followed by relaxation and a passive stretch. ${ }^{(6)}$

Muscle energy technique has been used to increase the flexibility, and ROM of a restricted joint. It has been proven that a single application of MET for GH joint horizontal abductors in baseball pitchers resulted in increased $\mathrm{GH}$ joint horizontal adduction and internal rotation. ${ }^{(7)}$

Similarly mulligan is proven to be effective in improving the various ranges of motions. Mulligan is widely used to treat various musculoskeletal dysfunctions. This approach has been found to be able to restore functional movements in joints, even after many years of restriction.

Mulligan is the concurrent application of pain free accessory mobilization with active or passive movement.

Thus, to assess the effect of Muscle energy technique vs Mulligan to improve Glenohumeral internal rotation deficit.

\section{MATERIALS AND METHODOLOGY}

Materials used:

- Goniometer

- Mulligan belt

- Plinth

- Towel

- Pen

- paper

\section{Study Design:}

- Type of study-Comparative

- Duration of study- 1 year

- Area-Metropolitan city

\section{Sample Design:}

- Sampling - Convenient

- Sample size- 30 (group A- 15, group B15)

- Sample population- under-19 cricket bowlers

\section{Inclusion criteria:}

- Age U-19 male cricket bowlers playing at district level.

- Bowlers participating in regular training in cricket were recommended.

- Subjects who are willing to participate in the study.

\section{Exclusion criteria:}

- Numbness or tingling in upper extremity 
- Previous shoulder or neck surgery

- Any systemic illness

- Shoulder conditions like adhesive capsulitis, labral tear, shoulder instability and rotator cuff injuries

- Cervical or spine pathologies

\section{PROCEDURE:}

30 cricket bowlers with presence of glenohumeral internal rotation deficit who were willing to participate in the study were included.

A written consent form was taken from the subjects in the language best understood by them.

Screening of the subject was done as per the inclusion and exclusion criteria.

The 30 participants were further divided into two groups A and B consisting of 15 subjects each.

Before application of muscle energy technique and mulligan active shoulder range of motion using full scale goniometer was taken.

All patients irrespective of what treatment they receive after the application of muscle energy technique or mulligan was given hot packs for relaxation and before treatment patients was asked to do stretching for internal rotation.

For muscle energy technique the participants were taken in supine position with shoulder and elbow in 90 degree abduction and flexion. Therapist had to move the humerus into internal rotation until first resistance is reached. Participants then had to perform a $5 \mathrm{sec}$ isometric contraction at approximately $25 \%$ of maximal effort in direction of external rotation against an opposing force provided by the therapist. $30 \mathrm{sec}$ active assisted stretch to the external rotators was then given by the therapist. Participants were instructed to relax and a new movement barrier was engaged.

For mulligan the participants were taken in a supine lying position with shoulder and elbow placed in 90 degree of flexion. Therapist then grasped the distal humerus with both the hands and mobilizing belt is secured around the therapist's waist and was perpendicular to the humerus. Therapists then pulled the mobilizing belt and shift its weight backwards to distract the joint laterally. Therapists sustains a lateral distracting force with belt meanwhile the patient performs internal rotation of the shoulder (actively). The program was continued for 4 weeks with 6 sessions per week. After 4 weeks the range of motion was checked.

\section{RESULTS AND ANALYSIS}

The data was collected and the normality test was done of the mean. The data passed the normality test. Then paired t-test was done to compare the pre and post value of both mulligan and muscle energy technique where both $\mathrm{p}$ value was found to be 0.0001 which is highly significant. The statistics were done using GraphPadPrism 9.0.0.

The subjects participated in the study were cricket bowlers. A comparative study was performed on 30 subjects, with the age group of under-19 years, out of which group A had 15 males and group B had 15 males.

\section{Group A}

The table shows the difference between the mean and standard deviation of pre and post mulligan value of cricket bowlers.

\begin{tabular}{|l|l|l|}
\hline Variables & Pre-value & Post-value \\
\hline Mean & 135.6 & 158.87 \\
\hline SD & 10.65 & 9.06 \\
\hline P-value & $<0.0001$ & $<0.0001$ \\
\hline
\end{tabular}

\section{Group B}

The table shows the difference between the mean and standard deviation of pre and post met value of cricket bowlers.

\begin{tabular}{|l|l|l|}
\hline Variables & Pre-value & Post-value \\
\hline Mean & 132.2 & 152.2 \\
\hline SD & 12.60 & 11.80 \\
\hline P-value & $<0.0001$ & $<0.0001$ \\
\hline
\end{tabular}




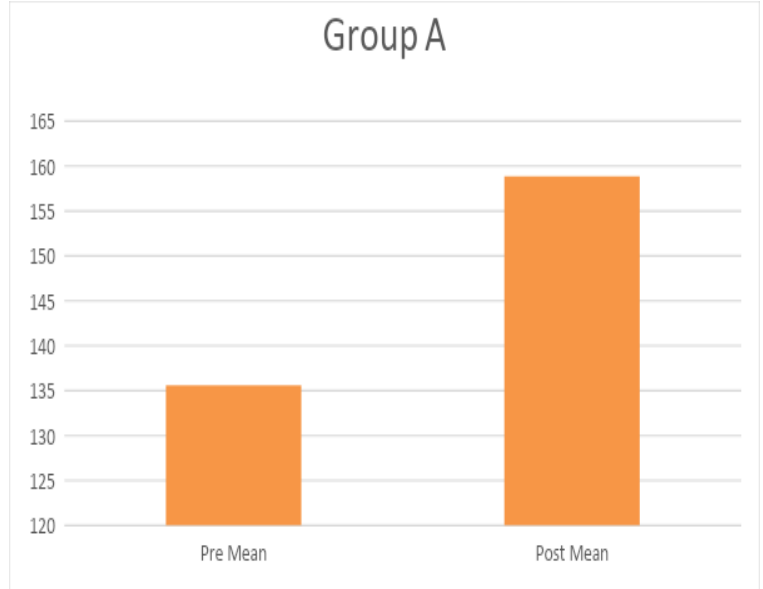

Graph 1: Representation of pre and post mean of mulligan (group A )

\section{Group B}

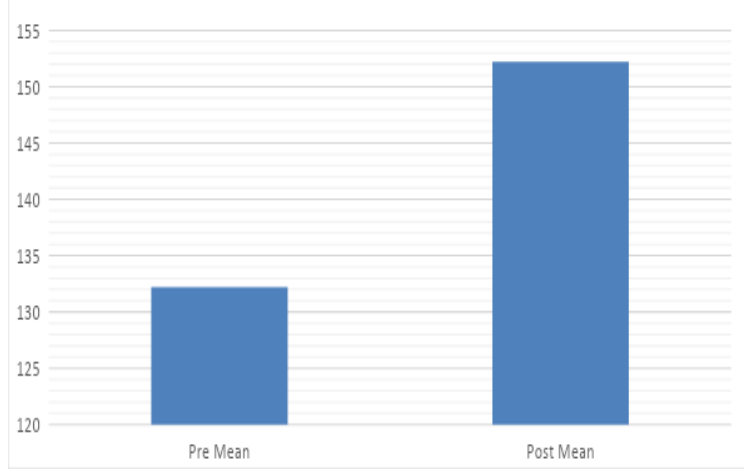

Graph 2: Representation of pre and post mean of MET (group B)

\section{DISCUSSION}

The present study was done to note the comparison between muscle energy technique and mulligan to improve GIRD in district level cricket bowlers. The study involved cricket bowlers of age U-19. The cricket bowlers included in the study on the basis of inclusion criteria such as bowlers participating in regular training in cricket and reduced total range of motion of dominant hand compared to non-dominant hand

Cricket bowlers place specific demands on the upper extremity, such as extreme velocities and high repetitions and often present with glenohumeral internal rotation deficit. Alterations to glenohumeral range of motion in the cricket bowlers have been linked to various pathologies including muscular dysfunction, impingement syndromes and labral lesions and also decreased performance. ${ }^{(8)}$

Brian Mulligan's mobilization with movement technique is widely used to treat various musculoskeletal dysfunctions. Mobilization with movement is a manual therapy technique based on the analysis and correction of any minor positional faults in a joint. Studies have shown that Mulligan techniques stretch the tightened soft tissues and also improve the normal extensibility of the shoulder capsule and normalize the abnormal scapulohumeral rhythm to induce beneficial effect. (9) Muscle energy techniques are a class of osteopathic manipulation methods that incorporate precisely directed and controlled, patient initiate isometric or isotonic contractions, designed to improve musculoskeletal function. MET may be used to decrease pain, stretch tight muscles and fascia, reduce muscle tonus, improve local circulation, strengthen weak musculature and mobilise joint restrictions. ${ }^{(8)}$

There is presence of GIRD in cricket bowlers, badminton players and non-player, with maximum in cricket bowlers. There is presence of injury in overhead athletes with GIRD, as well as rotational loss and external rotational gain. Further, mobilization techniques are support to increase or maintain joint mobility by including biological changes in synovial fluid. Mulligan technique stretches the tightened soft tissues and also improves the normal extensibility of the shoulder capsule. The effects of MET as decreased pain, increased range of motion, decreased muscle tension and spasm and increased strength. MET produced a change in range of motion was possibly due to an increased tolerance to stretch, as there was no evidence of viscoelastic change. MET also showed remarked improvement in the range of motion and strength of internal rotators in athletes with GIRD. ${ }^{(8)}$ Mulligan along with stretching exercises was more effective to patients as compared to mobilization with strengthening exercises given to patients with GIRD. 
Studies have brought individual effects of mulligan and MET for GIRD out in the light. But there are limited studies comparing mulligan and MET for cricket bowlers.

Hence the present study confirms the finding of different authors that both the mulligan and MET have almost similar effect on cricket bowlers with the presence of GIRD.

\section{CONCLUSION}

From the above study results, it is concluded that there is not much difference between Group A and group B when the values obtained were analysed. It indicated that group A mulligan had a significant improvement in range of motion in internal rotation and external rotation. It also indicated that group B MET also had a significant improvement in range of motion in internal rotation and external rotation. So it indicates that mulligan mobilization and met are effective in improving glenohumeral internal rotation deficit.

\section{ACKNOWLEDGEMENT}

I would like to show my greatest appreciation to respected sir Dr. Ajay Kumar. I am grateful to Dr. Deepali Rathod who helped me choosing my project topic and supported thoroughly on the way. Her willingness to motivate me contributed tremendously to my project. I also take this opportunity to thank my family members and friends for their help and support.

\section{Conflict of Interest: None}

\section{Source of Funding: None}

\section{Ethical Approval: Approved}

\section{REFERENCES}

1. Pardiwala DN, Rao NN, Varshney AV. Injuries in cricket. Sports health. 2018 May;10(3):217-22.

2. Sathya P, Parekh RN. Prevalence of Musculoskeletal Problems in Cricket
Players. Int J Health Sci Res. 2017; 7(8): 210-215.

3. Peter Brukner and Karim Khan. Clinical Sports Medicine. Third Edition

4. Rose MB, Noonan T. Glenohumeral internal rotation deficit in throwing athletes: current perspectives. Open access journal of sports medicine. 2018;9:69-78

5. Kibler WB, Sciascia A, Thomas SJ. Glenohumeral internal rotation deficit: pathogenesis and response to acute throwing. Sports medicine and arthroscopy review. 2012 Mar 1;20(1): 34-8

6. Chaitow L. Muscle energy techniques. Elsevier Health Sciences; 2013.

7. Moore SD, Laudner KG, Mcloda TA, Shaffer MA. The immediate effects of muscle energy technique on posterior shoulder tightness: a randomized controlled trial. journal of orthopaedic \& sports physical therapy. 2011 Jun;41(6):400-

8. Bathia K, Eapen C, Zulfeequer CP. Effectiveness of Muscle Energy Technique and Passive Stretching on Posterior Shoulder Tightness in Cricket Bowlers-A Randomized Clinical Trial. International Journal of Science and Research (IJSR). 2016;5(8):354-360

9. Kotagiri S, Songa AK, Gad MV, Lad J. Effectiveness of Mobilization with Exercise V/S Mulligan Internal Rotation MWM with Stretching in Patient with Glenohumeral Internal Rotation Deficit.

10. Bathia K, Sewani L, Sawantdesai P. Glenohumeral Internal Rotation Deficit (GIRD) in Asymptomatic Collegiate Cricket Bowlers and Badminton Players. Website: www. ijpot. com. 2017 Jul;11(3):51.

11. Sehgal S, Sen S, Dhawan A. Effects of Muscle Energy Technique in Increasing Range of Motion and Strength of Glenohumeral Internal Rotator, in Athletes with Glenohumeral Internal Rotation Deficit. American Journal of Sports Science. 2016;4(2):438 
Kimaya Vinod Kini et.al. Comparison of muscle energy technique vs mulligan to improve gird in district level cricket bowlers.

12. Borsa PA, Laudner KG, Sauers EL. Mobility and stability adaptations in the shoulder of the overhead athlete. Sports medicine. 2008 Jan 1;38(1):17-36.

13. Kibler WB, Chandler TJ, Livingston BP, Roetert EP. Shoulder range of motion in elite tennis players: effect of age and years of tournament play. The American journal of sports medicine. 1996 May; 24(3):279-85.

How to cite this article: Kini KV, Rathod D, Kumar A. Comparison of muscle energy technique vs mulligan to improve gird in district level cricket bowlers. Int $J$ Health Sci Res. 2021; 11(5): 88-93. DOI: https:// doi.org/10.52403/ijhsr.20210513

$* * * * * *$ 